\title{
Tsallis Entropy and the Vlasov-Poisson Equations
}

\author{
A. R. Plastino ${ }^{1,2,3}$ and A. Plastino ${ }^{3,4}$ \\ 1 Faculty of Astronomy and Geophysics, \\ National University La Plata, \\ C. C. 727, 1900 La Plata, Argentina \\ ${ }^{2}$ Centro Brasileiro de Pesquisas Físicas (CBPF) \\ Rua Xavier Sigaud 150, Rio de Janeiro, Brazil \\ 3 Argentine Research Agency (CONICET). \\ 4 Physics Department, \\ National University La Plata, \\ C.C. 727, 1900 La Plata, Argentina
}

Received 07 December, 1998

\begin{abstract}
We revisit Tsallis Maximum Entropy Solutions to the Vlasov-Poisson Equation describing gravitational $N$-body systems. We review their main characteristics and discuss their relationship with other applications of Tsallis statistics to systems with long range interactions. In the following considerations we shall be dealing with a $D$-dimensional space so as to be in a position to investigate possible dimensional dependences of Tsallis' parameter $q$. The particular and important case of the Schuster solution is studied in detail, and the pertinent Tsallis parameter $q$ is given as a function of the space dimension. In the special case of three dimensional space we recover the value $q=7 / 9$, that has already appeared in many applications of Tsallis' formalism involving long range forces.
\end{abstract}

\section{Introduction}

$$
S_{q}=\frac{1-\int[f(\mathbf{x})]^{q} d \mathbf{x}}{q-1}
$$

For a variety of physical reasons, much work has been devoted recently to the exploration of alternative or generalized information measures, based upon entropy functionals different from the standard BoltzmannGibbs-Shannon-Jaynes entropy [1]. Despite its great overall success, this orthodox formalism is unable to deal with a variety of interesting physical problems such as the thermodynamics of self-gravitating systems, some anomalous diffusion phenomena, Lévy flights and distributions, and turbulence, among others (see [1] for a more detailed list). The approach of Jaynes to Statistical Mechanics [2, 3] is also compatible with the possibility of building up a thermostatistics starting with a nonlogarithmic entropy, as demonstrated by Plastino and Plastino [4]. Tsallis has introduced a family of generalized entropies [5], namely, where $q$ is a real parameter characterizing the entropy functional $S_{q}$, and $f(\mathbf{x})$ is a probability distribution defined for $\mathbf{x} \in R^{N}$. In the limiting case $q \rightarrow 1$ the standard logarithmic entropy, $S_{1}=-\int f(\mathbf{x}) \ln f(\mathbf{x}) d \mathbf{x}$, is recovered. From an Information Theory viewpoint, (1) constitutes an information measure distinct from that of Shannon.

The Tsallis entropy preserves or suitable generalizes the relevant features of the Boltzamnn-Gibbs entropy $[5,6]$. It has been shown to be compatible with the Information Theory foundations of Statistical Mechanics given by Jaynes [4] and with the dynamical thermostating approach to statistical ensembles [7]. Increasing attention is being devoted to exploring the mathematical properties of Tsallis formalism [8-14,30].

The Tsallis formalism has been already applied to 
astrophysical self-gravitating systems [16, 17, 18], cosmology [19, 20], the solar neutrino problem [21], Lèvy flights and distributions [22, 23, 24], phonon-electron thermalization in solids [25], turbulence phenomena [26, 27], low-dimensional dissipative systems [28, 29], non linear Fokker-Planck equations [30], etc. The interested reader is referred to [1] for additional references.

Tsallis theory has also been confronted with direct experimental and observational data. Tsallis MaxEnt distributions have been shown to provide better descriptions than the ones given by the standard thermostatistics, for both the experimentally measured distributions of pure electron plasmas [26], and the observational peculiar velocity distribution of galaxy clusters [31]. Tsallis thermostatistics has also been advanced as a possible explanation for the solar neutrino problem [21]. It is worth remarking that all these experimental evidences involve $N$-body systems whose constituent particles interact according to the $r^{-1}$ gravitational (or Coulomb) potential.

Other alternative measures of entropy (or information) have also been successfully applied in different areas of theoretical physics. For instance, Rènyi information is a very useful tool in the study of chaotic dynamical systems [32]. However, it lacks a definite concavity, crucial for the discussion of thermodynamical stability. Tsallis entropy, on the other hand, does exhibit such an important mathematical property, and was thus historically the first one (besides the standard Boltzmann-Gibbs) employed to develop an entirely consistent Statistical Mechanics formalism [6, 4].

Active research conducted during the last few years is giving raise to a general picture of the physical scenarios where Tsallis theory provides a better thermostatistical description than the one given by the standard Boltzmann-Gibbs formalism. These physical systems are characterized by the presence of long range interactions, memory effects, or a fractal phase space. Summing up, they show nonextensive effects.
The first physical application of Tsallis entropy was concerned with self-gravitational systems [16]. It is well known that the standard Boltzmann-Gibbs formalism is unable to provide a useful description of this type of systems $[33,34,35,36]$. Moreover, the connection between Tsallis entropy and gravitation provided the first hint of just which are the kind of problems where the generalized formalism might be useful.

Although the standard Boltzmann-Gibbs formalism (characterized by the value $q=1$ of Tsallis parameter) has great difficulties in dealing with gravitation in three dimensional space, it shows no problems in the case of one or two dimensions. On the other hand, it is possible to obtain physically acceptable answers in $D=3$, if we employ an appropriate value of $q<7 / 9$. These facts suggest that dimensionality plays a decisive role within this context. The aim of the present work is to clarify some aspects of the application of Tsallis formalism to the Valsov-Poisson equations, and to generalize some of the known results connecting Tsallis Entropy with self-gravitating systems, in order to explore the possible dependence of the Tsallis parameter $q$ on the spatial dimensionality $D$.

\section{Gravitation and other Long Range Interactions.}

One of the main features characterizing the thermodynamics of self-gravitating systems is that the total energy, as well as other variables usually regarded as additive quantities, lose their extensive character. This fact suggests that a non-additive entropic measure, like the one proposed by Tsallis, may be more useful in dealing with gravitation than the standard Boltzamnn-Gibbs entropy. Indeed, non-extensivity is one of the main properties of Tsallis entropy. Given two non-correlated systems $A$ and $B$, with entropies $S_{q}[A]$ and $S_{q}[B]$, the Tsallis entropy $S_{q}[A+B]$ corresponding two both systems regarded as a single, unique, system is given by 


$$
S_{q}[A+B]=S_{q}[A]+S_{q}[B]+(1-q) S_{q}[A] S_{q}[B] .
$$

In the limit $q \rightarrow 1$, yielding Boltzmann-Gibbs thermostatistics, the standard additive behaviour of entropy is recovered.

In the case of gravitational physics, the nonextensive behaviour is due to the long range character of the gravitational interaction. The early applications of Tsallis' Thermostatistics to gravitational systems stimulated the exploration, within Tsallis formalism, of other many body problems with long range interaction showing non-extensive effects [37, 38, 39]. These studies considered systems with a Lennard-Jones like interparticle potential, characterized by a repulsive behaviour at short distances together with an attractive long range component falling like $r^{-\alpha}$. It is important to realize that this kind of potentials, while being useful in order to illuminate the thermodynamical consequences of long range forces, do differ in an essential way from the gravitational interaction. The Gibbs canonical ensemble for a system of a $N$ particles interacting via a Lennard-Jones-like potential, enclosed within a box of volume $V$, is well defined and has a convergent partition function. On the other hand, and due to the singularity at the origin of the gravitational potential, the Gibbs canonical ensemble for a system of $N$ gravitationally interacting particles (even if they are enclosed within a finite box) has a divergent partition function and thus is not well defined. The non-extensive properties of the Lennard-Jones like gases are more apparent when we try to define the $N \rightarrow \infty$ thermodynamic limit. The main results obtained so far in connection with this issue deal with the exotic scaling laws that are to be employed in orther to define the thermodynamic limit in a sensible way. Cogent evidence has already been obtained, showing that the correct scaling (with particle number) of the thermodynamic variables such as internal and free energy, is the one proposed by Tsallis, that goes with $N N^{*}$, where

$$
N^{*}=\frac{N^{(1-(\alpha / D))-1}}{1-(\alpha / D)},
$$

$D$ being the spatial dimension. But this scaling with the number of particles is not applicable to the strict gravitational case, since the canonical ensemble (for a given finite number of particles) is meaningless. There is another fundamental feature of the thermodynamics of systems endowed with long range interactions that has not yet been fully addressed by the simplified models discussed in $[37,38,39]$. This property is the breaking of the translational symmetry of space that is implicitly assumed in standard thermodynamics [40]. This property of systems with long range interactions is illustrated by the polytropic model discussed in [16] and reviewed here.

\section{The Vlasov-Poisson Equa- tions.}

Vlasov equation constitutes one of the most important mathematical tools employed to model astrophysical self-gravitating $N$-body systems like galaxies and galaxy clusters. Let us consider a system of $N$ identical stars of mass $m$. Vlasov equation reads [33]

$$
\frac{\partial f}{\partial t}+\mathbf{v} \cdot \frac{\partial f}{\partial \mathbf{x}}-\frac{\partial \phi}{\partial \mathbf{x}} \cdot \frac{\partial f}{\partial \mathbf{v}}=0
$$

where $f(\mathbf{x}, \mathbf{v}) d \mathbf{x} d \mathbf{v}$ denotes the number of stars in the $2 D$-dimensional volume element $d \mathbf{x} d \mathbf{v}$ in positionvelocity space, and the gravitational potential $\phi(\mathbf{x})$ is given by the $D$-dimensional Poisson equation

$$
\nabla^{2} \phi=D(D-2) V_{1} G \rho
$$

In the last equation $G$ is the universal gravitational constant, $V_{1}$ denotes the volume of a $D$-dimensional unitary sphere, and the mass density $\rho$ given by

$$
\rho(\mathbf{x})=m \int f(\mathbf{x}, \mathbf{v}) d \mathbf{v}
$$


In the particular case of a central potential $\phi(r)$ depending only on the radial coordinate $r$, the Laplacian operator adopts the form [41],

$$
\nabla^{2} \phi=\frac{1}{r^{D-1}} \frac{d}{d r}\left\{r^{D-1} \frac{d \phi}{d r}\right\}
$$

It is important to realize that Vlasov equation can be cast in the form

$$
\left(\frac{d f}{d t}\right)_{\text {orbit }}=0
$$

where the total time derivative is evaluated along the orbit of any star moving in the potential $\phi$. In the case of stationary solutions (i.e.; $\partial f / \partial t=0$ ), equation (8) implies that $f(\mathbf{x}, \mathbf{v})$ depends on the coordinates and the velocity components only through integration constants $C_{i}$ of the motion in the potential $\phi$. This result,

$$
f_{\text {est. }}=f\left(C_{1}, \ldots C_{N}\right)
$$

constitutes the well known Jeans' Theorem in Stellar Dynamics [33]. The solutions describing spherically symmetric systems with isotropic velocity distributions depend only on the energy, and it is convenient to write them in the form

$$
f=f(\epsilon)
$$

where

$$
\epsilon=\Phi(\mathbf{x})-\frac{1}{2} v^{2}
$$

and

$$
\Phi=\phi_{0}-\phi
$$

The quantities $\epsilon$ and $\Phi$ are usually called the relative energy and potential, respectively. The constant $\phi_{0}$ is chosen in such a way that the relative potential vanishes at the boundary of the system.

\section{MaxEnt Distributions in Stellar Dynamics}

Present day galaxies are described by stationary solutions to the Vlasov-Poisson equations. These equilibrium configurations are usually assumed to be the result of appropriate relaxation processes such as the ones denoted by the names "phase mixing" and "violent relaxation" [33]. These kind of processes are characterized by a loss of memory about the initial conditions of the system. It has been argued that the final equilibrium state should be determined from a MaxEnt variational principle [35]. The maximization of Boltzmann' logarithmic entropy under the constraints imposed by the conservation of the total mass and energy, leads to the stellar isothermal sphere distribution,

$$
f(\epsilon)=A\left(2 \pi \sigma^{2}\right)^{-3 / 2} \exp \left(\epsilon / \sigma^{2}\right)
$$

where $\sigma$ stands for the velocity dispersion and $A$ is an appropriate normalization constant. Unfortunately, the isothermal sphere is characterized by an infinite total mass [35]. The real meaning of this unphysical result is that the posed variational problem does not have a solution. For any given star distribution $f(\mathbf{x}, \mathbf{v})$, it is always possible to obtain a new distribution $f^{*}(\mathbf{x}, \mathbf{v})$ with the same mass and energy but showing a larger Boltzmann entropy [33]. For given values of total mass and energy, entropy is not bounded from above. An illustration of this feature of self-gravitating systems is provided by the so-called "red giant structure". This configuration is characterized by a high density inner core surrounded by a diluted extended "atmosphere". The core accounts for almost all the energy of the system. The outer envelope makes the main contribution to the total entropy. By increasing the concentration of the core while simultaneously expanding the "atmosphere", the entropy can be raised as much as wanted without changing the total energy. [33].

Summing up, a MaxEnt approach based on the 
Gibbs-Boltzmann entropic measure seems to be unable to characterize relaxed self-gravitating systems. This fact has motivated the exploration of alternative entropy functionals [36] of the form

$$
S=\int C[f] d \mathbf{x} d \mathbf{v},
$$

$C[f]$ being a convex function that vanishes for $f=0$.

Here enters Tsallis generalized entropy $S_{q}$. It has been shown that adopting appropriate values for Tsallis parameter $q$, the extremization of Tsallis functional $S_{q}$ under the constraints imposed by the total mass and energy leads to sensible stellar distributions. Indeed, the so-called stellar polytropes are obtained [16]. These distributions have been widely employed in the modelization of astrophysical objects like galaxies [33], although their relationship with Tsallis entropy was not known. The mass density of a stellar polytrope behaves in the same fashion as the density of a self-gravitating sphere constituted by a gas with a polytropic equation of state,

$$
p=\text { const. } \times \rho^{\gamma} .
$$

The exponent $\gamma$ in the equation of state is related to the polytropic index $n$ by

$$
\gamma=1+\frac{1}{n}
$$

Stellar polytropes constitute the most simple, but still physically acceptable, models for equilibrium stellar systems. In the earlier days of stellar dynamics, polytropes where considered as possible realistic models for galaxies and globular clusters. Nowadays it is known that stellar polytropes do not fit properly the concomitant observational data. However, they still play an important role in theoretical astrophysics, and are widely employed as a first theoretical approach, both in numerical simulations and analytical studies, for the description of stellar systems. From our point of view they are relevant because they illustrate how a generalized thermostatistical formalism, based on a non-extensive entropy, is able to deal in a physically sensible way with self-gravitating systems. Moreover, the present formalism is relevant in connection with many of the empirical verifications of Tsallis theory that we have up to now. Bogoshian's application of Tsallis entropy to the pure electron plasma [26] is based essentially in the same mathematical formalism as the one involved in the discussion of stellar polytropes. Furthermore, it has been recently shown [31] that a Tsallis distribution fits the observed velocity distribution of galaxy clusters in a much better way than other models based on the standard entropy. The reason for the success of Tsallis theory within this context, is presumably due to the long-range gravitational forces involved. And, so far, the stellar polytropes are the only detailed theoretical model establishing a link between Tsallis distributions and $N$-body self-gravitational systems. For all these reasons we believe that a further study of the relationship between stellar polytropes and Tsallis statistics is worthwhile, and will contribute to the understanding of non-extensive systems.

\section{Tsallis MaxEnt Solutions.}

Now we will extremize the Tsallis entropy of the star distribution function $f(\mathbf{x}, \mathbf{v})$,

$$
S_{q}=\frac{1}{q-1} \int\left(f-f^{q}\right) d \mathbf{x} d \mathbf{v},
$$

under the constraints imposed by the total mass

$$
\frac{M}{m}=\int f(\mathbf{x}, \mathbf{v}) d \mathbf{x} d \mathbf{v},
$$

and the total energy $E$,

$$
\frac{E}{m}=\frac{1}{2} \int f(\mathbf{x}, \mathbf{v})\left(v^{2}+\phi(\mathbf{x})\right) d \mathbf{x} d \mathbf{v} .
$$

In order to avoid some misunderstandings that arose in the literature in connection with this last formula [26], we must stress that the above expression deals with the 
system's gravitational potential in a self consistent way. The potential $\phi$ appearing in (19) is generated by the mass distribution $\rho$ associated with the distribution $f$ itself. We are not considering a given external potential. In such a case, the appropriate expression would be

$$
\frac{E^{*}}{m}=\int f(\mathbf{x}, \mathbf{v})\left(\frac{1}{2} v^{2}+\phi(\mathbf{x})\right) d \mathbf{x} d \mathbf{v} .
$$

Introducing now appropriate Lagrange multipliers $\lambda$ and $\beta$, our variational problem can be put in the form

$$
\delta\left(S_{q}-\beta \frac{E}{m}-\lambda \frac{M}{m}\right)=0,
$$

yielding the Tsallis MaxEnt solution

$$
\begin{aligned}
f_{M E} & =\left[\frac{1}{q}[1+(1-q) \lambda-\beta(q-1) e]\right]^{\frac{1}{q-1}} \\
& =\text { const } \times\left(\frac{\lambda(1-q)+1}{(q-1) \beta}-e\right)^{\frac{1}{q-1}},
\end{aligned}
$$

where

$$
e=\frac{1}{2} v^{2}+\phi
$$

is the energy (per unit mass) of an individual star.

Making the identification

$$
\phi_{0}=\frac{\lambda(1-q)+1}{(q-1) \beta}
$$

the MaxEnt distribution adopts the form of a stellar polytrope,

$$
f_{M E}=F \epsilon^{\alpha}
$$

with

$$
\alpha=\frac{1}{q-1},
$$

$F$ being constant. So far we have used, as the constraints in our variational approach, the ordinary kind of mean values. However, within Tsallis formalism, a generalization of the concept of mean value has been considered $[6,26]$. If the above calculations are re-done using Tsallis mean values, a politropic distribution is again obtained, but with a different value of the parameter $q$. This important point will be discussed in more detail in section (IV).

The cut-off condition on the generalized MaxEnt distribution was originally introduced by Tsallis in a rather ad-hoc way. However, within the present application of Tsallis generalized thermostatistics, the cutoff prescription admits of a clear physical interpretation. Within the polytrope stellar distributions, the Tsallis cut-off corresponds to the escape velocity from the system [33].

The mass density distribution associated with the politropic distribution is

$$
\rho(\mathbf{x})=D V_{1} F \int_{0}^{\sqrt{2 \Phi}}\left(\Phi-\frac{1}{2} v^{2}\right)^{\alpha} v^{D-1} d v
$$

This expression can be simplified making the change of variables

$$
v^{2}=2 \Phi \cos ^{2} \theta,
$$

wich yields

$$
\rho=2^{D / 2} D V_{1} F \Phi^{\alpha+D / 2} \int_{0}^{\pi / 2} \sin ^{2 \alpha+1} \theta \cos ^{D-1} \theta d \theta .
$$

Introducing now the politropic index 


$$
n=\alpha+\frac{D}{2}
$$

we obtain

$$
\rho=2^{D / 2} D V_{1} F \Phi^{n} \int_{0}^{\pi / 2} \sin ^{2 n-D+1} \theta \cos ^{D-1} \theta d \theta .
$$

This last equation can be recast in the simpler form

$$
\rho=C \Phi^{n},
$$

where $C$ is now a constant given by

$$
C=2^{D / 2} D V_{1} F \int_{0}^{\pi / 2} \sin ^{2 n-D+1} \theta \cos ^{D-1} \theta d \theta .
$$

Inserting this expression in the $D$-dimensional Poisson equation (5) we obtain a non-linear ordinary differential equation for $\Phi$ (recall that $\Phi=\phi_{0}-\phi$ ),

$$
\frac{1}{r^{D-1}} \frac{d}{d r}\left[r^{D-1} \frac{d \Phi}{d r}\right]+D(D-2) V_{1} G C \Phi^{n}=0 .
$$

The expression for the mass density $\rho$ also allows us to obtain the radial derivative of the total mass $M(r)$ contained within radious $r$,

$$
\frac{d M}{d r}=D V_{1} r^{D-1} \rho=D V_{1} r^{D-1} C \Phi^{n} .
$$

Inserting this last result in the differential equation verified by $\Phi$, it is easy to see that

$$
\left[r^{D-1} \frac{d \Phi}{d r}\right]+G(D-2) M(r)=\text { const }
$$

which evaluated in $r=0$ leads to

$$
\left[r^{D-1} \frac{d \Phi}{d r}\right]+G(D-2) M(r)=0 .
$$

From this last equation it follows that $\Phi \sim r^{2-D}$ is the limiting asimptotic behaviour still yielding a finite total mass.

\section{D-dimensional Spheres.}

In terms of the dimensionless variables

\section{Schuster the differential equation for $\Phi$ reads}

$$
h=\Phi / \Phi_{0},
$$

and

$$
\frac{1}{y^{D-1}} \frac{d}{d y}\left[y^{D-1} \frac{d h}{d y}\right]+h^{n}=0
$$

If we adopt the politropic index

$$
n=\frac{D+2}{D-2},
$$

$$
y=\left[D(D-2) V_{1} G C \Phi_{0}^{n-1}\right]^{1 / 2} r,
$$

the above equation admits the solution 


$$
h(y)=\left[1+\frac{y^{2}}{D(D-2)}\right]^{\frac{2-D}{2}},
$$

which, in term of the physical variables reads,

$$
\Phi=\Phi_{0}\left[1+\frac{j^{2} r^{2}}{D(D-2)}\right]^{\frac{2-D}{2}}
$$

where

$$
j=\left[D(D-2) V_{1} G C \Phi_{0}^{n-1}\right]^{1 / 2} .
$$

From equation (37) it follows that the $D$ dimensional Schuster Sphere shows the limiting asimptotic behaviour for a finite total mass.

The exponent $\alpha$ appearing in the stellar politrope distribution is now

$$
\alpha=n-\frac{D}{2}=\frac{8-(D-2)^{2}}{2(D-2)} .
$$

Notice that for a space dimension $D>D_{l}$, where

$$
D_{l}=2+2 \sqrt{2},
$$

the $D$-dimensional Schuster politrope is a monotonic increasing function of the energy.

\section{Tsallis generalized Mean Values}

Tsallis generalized mean values constitute a remarckable component of the generalized thermostatistical formalism that has not appeared yet in the present considerations. Given a probability distribution $f$, the generalized mean value of a quantity $A$ is defined as

$$
\langle A\rangle_{q}=\int A f^{q} d \Omega
$$

where $d \Omega$ stands for the volume element on the appropriate phase space where the distribution $f$ is defined.
The physical interpretation of the $q$-generalized mean values poses a difficult problem. For instance, we have to deal with the baffling fact that the mean value of 1 is not equal to 1 . The original motivation for introducing the generalized mean values was of a rather formal nature. Tsallis and Curado proved that, if we replace the standard mean values by the generalized ones, all the usual thermodynamical relations are preserved within Tsallis formalism [6]. We now know, however, that the connection with thermodynamics still holds true if we employ Tsallis entropy along with the standard mean values [43, 44]. This last result somewhat weakened the case for the $q$-generalized mean values. On the other hand, the generalized mean values show some desirable properties in connection with thermodynamic stability. Another advantage of the $q$-values is that they are useful in order to characterize distributions with divergent (ordinary) moments. For instance, $\left\langle x^{2}\right\rangle$ is divergent for Lévy distributions while, employing an appropriate Tsallis parameter $q$ different from unity, $\left\langle x^{2}\right\rangle_{q}$ converges.

The first discussion of Vlasov-Poisson dynamics in connection with Tsallis statistics was done employing the usual linear mean values [16]. Afterwards, Boghosian [26] reformulated those results in terms of the $q$-generalized mean values. In order to appreciate Boghosian' contribution, it is useful to consider the following expression for the kinetical and gravitational potential energies of the system

$$
T=m \int \frac{1}{2} v^{2} f(\mathbf{r}, \mathbf{v}) d \mathbf{r} d \mathbf{v}
$$

and

$$
\begin{aligned}
W & =-G \iint \frac{\rho\left(\mathbf{r}_{\mathbf{1}}\right) \rho\left(\mathbf{r}_{\mathbf{2}}\right)}{\left|\mathbf{r}_{\mathbf{1}}-\mathbf{r}_{\mathbf{2}}\right|^{D-2}} d \mathbf{r}_{\mathbf{1}} d \mathbf{r}_{\mathbf{2}} \\
& =-G m^{2} \iint \frac{f\left(\mathbf{r}_{\mathbf{1}}, \mathbf{v}_{\mathbf{1}}\right) f\left(\mathbf{r}_{\mathbf{2}}, \mathbf{v}_{\mathbf{2}}\right)}{\left|\mathbf{r}_{\mathbf{1}}-\mathbf{r}_{\mathbf{2}}\right|^{D-2}} d \mathbf{r}_{\mathbf{1}} d \mathbf{v}_{\mathbf{1}} d \mathbf{r}_{\mathbf{2}} d \mathbf{v}_{\mathbf{2}}
\end{aligned}
$$


In order to introduce Tsallis generalized mean values, Boghosian defined the following generalizations for the kinetic and potential energies,

$$
T_{q}=m \int \frac{1}{2} v^{2} f^{q}(\mathbf{r}, \mathbf{v}) d \mathbf{r} d \mathbf{v}
$$

and

$$
W_{q}=-G m^{2} \iint \frac{f^{q}\left(\mathbf{r}_{\mathbf{1}}, \mathbf{v}_{\mathbf{1}}\right) f^{q}\left(\mathbf{r}_{\mathbf{2}}, \mathbf{v}_{\mathbf{2}}\right)}{\left|\mathbf{r}_{\mathbf{1}}-\mathbf{r}_{\mathbf{2}}\right|^{D-2}} d \mathbf{r}_{\mathbf{1}} d \mathbf{v}_{\mathbf{1}} d \mathbf{r}_{\mathbf{2}} d \mathbf{v}_{\mathbf{2}}
$$

Moreover, he proposed to identify the mass density with

$$
\rho(\mathbf{r})=m \int f^{q}(\mathbf{r}, \mathbf{v}) d \mathbf{v},
$$

Boghosian' approach is tantamount to identify the actual stellar distribution with $f^{q}$ instead of making the identification with $f$ itself. Consequently, a Tsallis distribution with parameter $q$, obtained by recourse to the usual mean values, is equivalent to a Tsallis distribution a la Boghosian with a new parameter $q^{*}$ given by

$$
\frac{q^{*}}{1-q^{*}}=\frac{1}{q-1} .
$$

Hence, making use of the complete Tsallis formalism, the Tsallis parameter characterizing the $D$-dimensional Schuster distributions is given by

$$
q^{*}=\frac{8-(D-2)^{2}}{8-(D-2)^{2}+2(D-2)} .
$$

It is interesting to notice that in the limit $D \rightarrow 2$, we obtein $q^{*} \rightarrow 1$. This is consistent with the known fact that the standard Boltzmann-Gibbs Thermostatistics is able to deal in a physically acceptable way with selfgravitating systems with spatial dimension less than 2 $[34,42]$. In the case of $D=3$, we recover the known value $q^{*}=7 / 9[26]$ corresponding to the limit value of $q^{*}$ providing a polytropic distribution with finit total mass.

A different kind of generalized mean values have been recently introduced by Tsallis and coworkers $[45,46]$. These mean values are defined by

$$
\langle\langle A\rangle\rangle_{q}=\frac{\int f^{q} A d \Omega}{\int f^{q} d \Omega}
$$

where $d \Omega$ is the volume element in the relevant phase space where the distribution $f$ "lives". These generalized mean values still do exhibit the convenient properties of Tsallis' original q-moments. However, the new normalized mean values are free of many of the troublesome features of the original $q$-values. For instance, we now recover the desirable relation

$$
\langle\langle 1\rangle\rangle_{q}=1 .
$$

It would be interesting to reformulate the $q$ nonextensive approach to stellar dynamics in terms of the normalized $q$-mean values (55). When the normalized $q$-values are used, the nonextensive $q$ thermostatistics can be reformulated in terms of ordinary linear mean values [46]. This fact suggests that this new formulation of Tsallis formalism may be particularly useful in order to develope the $q$-nonextensive version of the BBGKY hierarchy [33]. This line of work poses formidable difficulties when the unnormalized $q$ mean values are used.

\section{The $q=-1$ MaxEnt Time Dependent Solutions to the Vlasov- Poisson Equa- tions}

The Tsallis nonextensive thermostatistics is also useful in order to obtain approximate time dependent solutions to the Poisson-Vlasov equations [17]. The $q$-MaxEnt 
scheme developed in [17] is based on the idea of follow-

of $M$ relevant dynamical quantities $A_{i}(\mathbf{x}, \mathbf{v})$, ing the time evolution of the mean values of a small set

$$
\left\langle A_{i}\right\rangle_{q}=\frac{1}{h} \int f^{q}(\mathbf{x}, \mathbf{v}) A_{i}(\mathbf{x}, \mathbf{v}) d \mathbf{x} d \mathbf{v} \quad(i=1, \ldots, M),
$$

where $h$ is just a constant with the dimensions of the element of volume in the pertinent $(\mathbf{x}, \mathbf{v})$ space. If the distribution function $f(\mathbf{x}, \mathbf{v})$ evolves according to the Vlasov equation the time derivative of the relevant mean values is given by

$$
\frac{d}{d t}\left\langle A_{i}\right\rangle_{q}=\frac{1}{h} \int f^{q}(\mathbf{x}, \mathbf{v})\left(\mathbf{v} \cdot \nabla_{x} A_{i}-\nabla \phi \cdot \nabla_{v} A_{i}\right) d \mathbf{x} d \mathbf{v} \quad(i=1, \ldots, M) .
$$

Unfortunatelly, this last set of equations does not constitute, in general, a closed set of $M$ ordinary differential equations for the mean values $\left\langle A_{i}\right\rangle_{q}$. However, the MaxEnt approach allows us to build up, at each time $t$, a $q$-MaxEnt distribution

$$
f_{q}(\mathbf{x}, \mathbf{v}, t)=\frac{1}{Z_{q}}\left[1-(1-q) \sum_{i=1}^{M} \lambda_{i}(t) A_{i}(\mathbf{x}, \mathbf{v})\right]^{1 /(1-q)}
$$

taking as constraints the instantaneous values adopted by the relevant moments $\left\langle A_{i}\right\rangle_{q}$ and introducing appropriate Lagrange multipliers $\lambda_{i}(i=1, \ldots, M)$. The right hand sides of equations (58) can then be evaluated by recourse to this $q$-MaxEnt approximation. In such a manner a closed system of equations is formally obtained. The ensuing system, however, becomes highly non-linear. As a counterpart, the Vlasov equation, a partial differential equation, becomes now a system of ordinary differential equations for the evolution of the relevant mean values. In [17] it has been shown that such an approach is indeed useful because it does preserve some important properties of the exact evolution equation.

The $q$-MaxEnt approxiamte solution $f_{q}(\mathbf{x}, \mathbf{v}, t)$ preserves, by construction, the exact equations of motion of our $M$ relevant moments. These moments, along with the concomitant Lagrange multipliers, evolve according to a set of $2 M$ ordinary differential equations that can be given a Hamiltonian form [17]: the relevant mean values $\left\langle A_{i}\right\rangle_{q}$ and their associated Lagrange mul- tipliers turn out to be canonicaly conjugate dynamical variables. Due to this Hamiltonian structure the associated flow in phase space does not have sinks, nor does it have sources. Moreover, the Tsallis entropy $S_{q}$ evaluated upon the MaxEnt approximate solution $f_{q}(\mathbf{x}, \mathbf{v})$ is a constant of the motion. This means that the evolution of the system does not exhibit any relaxation process. This is an important feature of the exact solutions that is shared by our MaxEnt approximations. All these general properties of the $q$-MaxEnt scheme do not depend on the dimensionality of the (one-particle) configuration space. They hold true for any number of spatial dimensions. However, in the particular case of $q=-1$ and $D=1$ or $D=3$, our MaxEnt approximation generates, if the relevant mean values are properly chosen, exact solutions to the Vlasov-Poisson equations [17]. Indeed, various of the known exact solutions of the Valsov-Poisson equations turn out to be particular instances of our general $q$-MaxEnt approach [17].

It should be stressed that these time dependent $q$ - 
MaxEnt solutions of the Vlasov equation are not related in a direct fashion to the previously discussed stationary MaxEnt solutions. The total energy does not appear as a constraint within the time dependent scenario, as happens in the stationary case. This means that the $q=-1$ value of the Tsallis parameter leading to exact time dependent solutions should not be regarded as belonging to the "allowed" $q<7 / 9$ range of $q$-values yielding physically acceptable stationary solutions. These two special $q$-values, $7 / 9$ and -1 , arise from quite different circumstances and, as far as we know, are completely unrelated.

\section{Conclusions}

We have considered MaxEnt Tsallis distributions in connection with stationary solutions of the $D$ dimensional Vlasov-Poisson equations. In particular, we obtained an analitical relation between Tsallis parameter $q$ and the dimension $D$ of physical space for the special case of $D$-dimensional Schuster solutions. Although these solutions have infinite spacial extent, the density falls rapidly enough so as to have a finite total mass. Furthermore, they show, within the larger set of Tsallis MaxEnt solutions, the limiting behaviour between sensible solutions with finite mass and unphysical solutions with divergent mass.

\section{Acknowledgments}

A. R. Plastino gratefully acknowledges the hospitality of the Centro Brasileiro de Pesquisas Físicas (CBPF), where part of this research was done.

\section{References}

[1] C. Tsallis, Physica A221, 277 (1995); C. Tsallis, Physics World 10, 42 (July 1997).

See http://tsallis.cat.cbpf.br/biblio.htm for a complete and updated bibliography.

[2] E. T. Jaynes in Statistical Physics, ed. W. K. Ford (Benjamin, New York, 1963); A. Katz, Statistical Mechanics, (Freeman, San Francisco, 1967).
[3] J. Aliaga, D. Otero, A. Plastino and A. N. Proto, Phys. Rev. A37, 918 (1988); E. Duering, D. Otero, A. Plastino and A. N. Proto, Phys. Rev. A32, 2455 (1985); E. Duering, D. Otero, A. Plastino and A. N. Proto, Phys. Rev. A35, 2314 (1987); A. R. Plastino and A. Plastino, Physica A232, 458 (1996).

[4] A. R. Plastino and A. Plastino, Phys. Lett. A177, 177 (1993).

[5] C. Tsallis, J. Stat. Phys. 52, 479 (1988).

[6] E. M. F. Curado and C. Tsallis, J. Phys. A24, L69 (1991); Corrigenda: 24, 3187 (1991) and 25, 1019 (1992).

[7] A. R. Plastino and C. Anteneodo, Ann. Phys., 255, 250 (1997).

[8] G. A. Raggio, J. Math. Phys. 36, 4785 (1995).

[9] G. R. Guerberoff and G. A. Raggio, J. Math. Phys. 37, 1776 (1996).

[10] G. R. Guerberoff, P. A. Pury and G. A. Raggio, J. Math. Phys. 37, 1790 (1996).

[11] A. M. Meson and F. Vericat, J. Math. Phys. 37, 4480 (1996).

[12] A. K. Rajagopal, Phys. Rev. Lett. 76, 3469 (1996).

[13] E. K. Lenzi, L. C. Malacarne and R. S. Mendez, Phys. Rev. Lett. 80, 218 (1997).

[14] S. Abe, Phys. Lett. A 224, 326 (1997).

[15] E. P. Borges, J. Phys. A: Math. Gen. 31, 5281 (1998).

[16] A. R. Plastino and A. Plastino, Phys. Lett. A174, 384 (1993).

[17] A. R. Plastino and A. Plastino, Phys. Lett. A193, 251 (1994).

[18] J.J. Aly, in "N-Body Problems and Gravitational Dynamics", Proc. of the Meeting held at Aussois-France (21-25 March 1993), eds. F. Combes and E. Athanassoula (Publications de l'Observatoire de Paris, 1993).

[19] V.H. Hamity and D.E. Barraco, Phys. Rev. Lett. 76, 4664 (1996).

[20] D.F. Torres, H. Vucetich, and A. Plastino, Phys. Rev. Lett. 79, 1588 (1997).

[21] G. Kaniadakis, A. Lavagno and P. Quarati, Phys. Lett. B 369, 308 (1996).

[22] P. A. Alemany and D. H. Zanette, Phys. Rev. E49, 956 (1994).

[23] D. H. Zanette and P. A. Alemany, Phys. Rev. Lett. 75, 366 (1995).

[24] C. Tsallis, S. V. F. Levy, A. M. C. Souza and R. Maynard, Phys. Rev. Lett. 75, 3589 (1995).

[25] I. Koponen, Phys. Rev. E55, 7759 (1997).

[26] B. M. Boghosian, Phys. Rev. E53, 4754 (1995).

[27] C. Anteneodo and C. Tsallis, J. Mol. Liq. 71, 255 (1997).

[28] C. Tsallis, A. R. Plastino and W.-M. Zheng, Chaos, Solitons, and Fractals 8, 885 (1997). 
[29] M. L. Lyra and C. Tsallis, Phys. Rev. Lett., 80, 53 (1998).

[30] L. Borland, Phys. Rev. E 57, 6634 (1998).

[31] A. Lavagno, G. Kaniadakis, M. Rego-Monteiro, P. Quarati and C. Tsallis, Nonextensive Thermostatistical Approach to the Peculiar Velocity Function of Galaxy Clusters, Astrophysical Letters and Communications, 35, 449 (1998).

[32] C. Beck and F. Schloegl, Thermodynamics of chaotic systems, (Cambridge UP, Cambridge, 1993).

[33] J. Binney and S. Tremaine, Galactic Dynamics, (Princeton U.P., Princeton, 1987).

[34] W. C. Saslaw, Gravitational Physics of Stellar and Galactic Systems, (Cambridge University Press, Cambridge, 1985).

[35] D. Lynden-Bell, Mon. Not. R. Astron. Soc. 124 (1967) 279.

[36] S. Tremaine, M. Henon and D. Lynden-Bell, Mon. Not. R. Astron. Soc. 219 (1986) 285.

[37] P. Jund, S.G. Kim and C. Tsallis, Phys. Rev. B52 (1995) 50.
[38] L. S. Lucena, L. R. da Silva and C. Tsallis Phys. Rev. E51, (1995) 6247.

[39] J. R. Grigera, Phys. Lett. A217, (1996) 47.

[40] J. D. Brown and J. W. York Jr., in Physiscal Origins of Time Assymetry, (J. J. Halliwell, J. Perez-Mercader, and W. H. Zurek (Editors), Cambridge Univ. Press., Cambridge, 1996) pp. 465-474.

[41] P. M. Morse and H. Feshbach, Methods of Theoretical Physics, (McGraw-Hilll, New York, 1953).

[42] G. Rybicki, Astrophys. and Space Sci. 14 (1971) 56.

[43] A. Plastino and A. R. Plastino, Phys. Lett. A 226, 257 (1997).

[44] R. S. Mendes, Physica A 242, 299 (1997).

[45] C. Tsallis, R. S. Mendes and A. R. Plastino, Role of the Constraints Within Generalized Nonextensive Statistics, Physica A 261, 534 (1998).

[46] R. P. Di Sisto, S. Martínez, R. B. Orellana, A. R. Plastino, and A. Plastino, General Thermostatistical Formalisms, Invariance Under Uniform Spectrum Translations, and Tsallis $q$-Additivity, (1998) Physica A, in press. 